\title{
Bayesian Network and Hidden Markov Model for Estimating occupancy from measurements and knowledge
}

\author{
Manar AMAYRI ${ }^{1}$, Quoc-Dung Ngo ${ }^{2}$, EL Abed EL Safadi ${ }^{3}$, Stephane Ploix ${ }^{4}$ \\ ${ }^{1}$ G-SCOP laboratory / Grenoble Institute of Technology, e-mail: Manar.Amayri@grenoble-inp.fr. \\ 2 People's Security Academy of Hanoi, Vietnam \\ ${ }^{3}$ Grenoble Alpes University(UGA) \\ ${ }^{4}$ G-SCOP laboratory / Grenoble Institute of Technology 46 Avenue Felix Viallet, 38031 Grenoble, France
}

\begin{abstract}
A general approach is proposed to determine the occupancy in a room using sensor data and knowledge coming respectively from observation and questioning are determined. Means to estimate occupancy include motion detections, power consumptions and acoustic pressure rewarded by a microphone. The proposed approach is inspired from machine learning. It starts by determining the most useful measurements in calculating information gains. Then, a non supervised estimation algorithm is proposed: it relies on Hidden Markov Model and Bayesian Network algorithms to model a human behaviour with probabilistic cause-effect relations and states based on knowledge and questioning. Bayesian Network(BN) and Hidden Markov Model (HMM) based approaches have been applied to an office setting, with an average estimation error of 0.1-0.08 and an accuracy of 89\%- $91 \%$. This approach avoids the usage of a camera to determine the actual occupancy required for supervised learning.
\end{abstract}

Keywords - Bayesian methods, Learning theory, Sensor networks, Knowledge modelling and knowledge based systems, Artificial intelligence.

\section{INTRODUCTION}

Recently, research about building turns to focus on occupant behavior: most of the world standards for buildings take occupants into account with representative values and deterministic scenarios: number of occupants, predefined schedules, and responses to an exceeded threshold. Non supervised learning machine (i.e., Hidden Markov, bayesian networks......) can collect knowledge from occupants through answers to some proposed questions or give observation information in order to build a knowledge structure to estimate the number of occupants in a studied area.

It is an important challenge in occupancy estimation because it solves the issue of the measurement of actual occupancy, usually done thanks to cameras and a posteriori labeling, which are both time consuming and invasive.

The authors acknowledge the support of the French Agence Nationale de la Recherche (ANR) under reference ANR-13-VBDU-0006 (OMEGA project)
Most of the works deal with the design stage: the target is to represent the diversity of occupant behavior in order to improve building energy management capabilities. Most of the approaches use statistics about human behavior [1]-[3]. [4] emphasized that inhabitants' detailed reactive and deliberative behavior must also be taken into account and proposed a co-simulation methodology to find out the impact of certain actions on energy consumption. Nevertheless, human behavior is not only interesting during the design step, but also during operation. It is indeed useful for performance guarantee and for diagnostic purposes to discriminate human misbehavior from building system poor performance, and also for energy management where strategies depend on human activities and, in particular, on the number, of occupants in a zone. Such a system has to be trained in each new environment. Unfortunately, using supervised learning algorithm on site is not widely accepted because of the required target to build the set of training data, which usually come from cameras which are not acceptable for many users. In addition, labeling occupancy from videos is time consuming. This paper tackles this issue. It proposes an occupancy estimation approach based on the human knowledge and questioning the occupants in the studied area. Section II presents a state of the art about occupancy estimation. Section III discusses the problem statement of occupancy estimation in supervised learning algorithm (i.e, Decision tree.....) Section IV propose a process for occupancy estimations using alternatively Hidden Markov Model (HMM) and Bayesian network (BN) knowledge. Section $\mathrm{V}$ propose structures for $\mathrm{HMM}$ and $\mathrm{BN}$, and section VI analyses the resulting occupancy estimations for an office.

\section{STATE OF THE ART}

In the scientific community of building physics, there is a growing interest for occupant behaviour because of its importance for energy waste reduction in buildings [5]. The occupant behaviour may be studied from building 
physics to human biology, through sociology and psychology in order to model and assess thermal and visual comfort, indoor air quality, etc. [6] uses neural networks for the agents to learn their behaviour from recorded data to ensure their comfort. After a learning phase, agents know the actions that increase their comfort in different environmental conditions. In a recent study, [7] models various occupancy profiles by calibration to predict the use of fans, heating systems and windows.

This paper focuses on estimation of occupancy but results could be easily extended to activity estimations.

Hidden Markov Model (HMM) and its variants have been extensively studied and utilized quite often to detect activities in the past. [8] utilizes an extension, Layered $H M M s$ in their model SEER, to detect various activities like desk work, phone conversations, presence, etc.... The layered structure of their model makes it feasible to decouple different levels of analysis for training and inference. Each level in the hierarchy can be trained independently, with different feature vectors and time granularities. Once the system has been trained, inference can be carried out at any level of the hierarchy. One benefit of such a model is that each layer can be trained individually in isolation, and therefore the lowest layer which is most sensitive to environment noises and flickers, can be retrained without touching the upper layers.

Different approaches for estimating occupancy have been investigated. Methods vary from basic single feature classifiers that distinguish among two classes (presence and absence) to multi-sensor, multi-feature models. A primary approach, which is prevalent in many commercial buildings, is the usage of passive infrared (PIR) sensors for occupancy. However, motion detectors fail to detect a presence when occupants remain relatively still, which is quite common during activities like working on a computer or regular desk work. This makes the use of only PIR sensors for occupancy counting purpose less attractive. Conjunction of PIR sensors with other sensors can be useful as discussed in [10]. It uses motion sensors and magnetic reed switches for occupancy detection to increase the efficiency of HVAC systems in smart buildings, which is quite simple and non-intrusive. Apart from motion, counting acoustic sensors may be used. However, audio from the environment can easily fool such sensors, and with no support from other sensors, it can report many false positives. In the same way, other sensors like video cameras and computer vision [9]. RFID tags [11] installed on id cards, sonar sensors [12] plugged on monitors to identify presence of a person on a computer, have been used and have proved to be much better at solving the problem of occupancy counting, which can not be employed in most office buildings for reasons like privacy and cost concerns. The use of pressure and PIR sensors to determine presence/absence in single desk offices has been discussed in [13]; it further tags activities based on this knowledge. However, for various applications like activity recognition or context analysis within a larger office space, information regarding the presence or absence of people is not sufficient and an estimation of the number of people occupying the space is essential. [14] investigates this problem in open offices, estimating occupancy and human activities using a multitude of ambient information, and compare the performance of HMMs, SVMs and Artificial Neural Networks. However, none of these methods generate human-understandable rules which may be very helpful to building managers.

In general, an occupancy counting algorithm that fully exploits information available from low cost, nonintrusive environmental sensors and knowledge to provide meaningful estimating is an important yet little explored problem in office buildings. This occupancy detection systems still have certain limitations with respect to occupant privacy.

\section{Problem Statement}

\section{A. Test bed}

The test bed is an office in Grenoble Institute of Technology, which accommodates four persons. The office has frequent visitors with a lot of meetings and presentations all through the week. The set-up for the sensor network includes:

1) An ambiance sensing network, which measures luminance, temperature, relative humidity $(\mathrm{RH})$, motions, CO2 concentration, power consumption, door and window position, microphone. Data are sent thanks to ENOCEAN protocol on significant value change event.

2) 2 video cameras for recording real occupancies and activities. Those two cameras are only used for validation purpose.

3) A centralized database with a web-application for retrieving data from different sources continuously.

To estimate the number of occupants, a relation has to be discovered between the office environment and the number of people in it. The office environment can be represented as a set of state variables, $A_{t}=$ $\left[A_{1}, A_{2}, \ldots, A_{m}\right]_{t}$. This set of state variables $A$ at any instance of time $t$ must be indicative of occupancy. A state variable can be termed as a feature, and therefore the set of features as feature vector. Similarly, the m-dimensional space that contains all possible values of such a feature vector is the feature space. The underlying approach for the experiments is to formulate the classification problem as a maping from a feature vector into some feature space that comprises several classes of occupancy or activity. Therefore, the success of such an approach heavily depends on how good the selected features are. In this case, features are attributes from multiple sensors accumulated over a time interval. The choice of interval duration is highly context dependent, and has to be done according 
to the required granularity. However, some features do not allow this duration to be arbitrarily small. As an example, it has been observed that $\mathrm{CO}_{2}$ levels do not rise immediately, and one of the factors affecting this delay is the ventilation of the space being observed. The results presented in this paper are based on an interval of $T_{s}=$ 30 minutes (which has been referred here to 1 quantum).

Features are the information extracted from the data i.e acoustic pressure from a microphone, time slot, occupancy from power consumption, door or window position, motion counting, day type, indoor temperature,... One quantitative measurement of the usefulness of a feature is information gain, which depends on the concept of entropy [15]. Information gain is helpful to distinguish among a large set of features, the most worthwhile to consider for occupancy estimation. A supervised learning approach has been used. Occupancy has been measured before using a classification algorithm: occupancy counting was manually annotated using a video feed from two cameras strategically positioned in an office. A decision tree algorithm has been used because it is results human readable, can be adjusted using expert knowledge and yield estimation rules (if-then) from the decision tree structure.

From the large set of features displayed in [15], some of them may not be worthwhile to consider, to achieve our target of occupancy classification. After removing less important features, the following main features were considered:

\begin{tabular}{cc} 
& Table I. BASIC SET OF FEATURES \\
1 & motion counter \\
2 & acoustic pressure from microphone \\
3 & occupancy from power consumption \\
\hline
\end{tabular}

They will be used in the next experiments of occupants estimation using the non supervised learning machine.

\section{PROPOSED GENERAL PROCESS FOR OCCUPANTS ESTIMATION}

\section{A. Bayesian Network}

A Bayesian Network (BN) offers compact and intuitive graphical representations of the uncertain relations among the random variables in a domain and their conditional dependencies via a directed acyclic graph. It is a knowledge model using conditional probabilities and evidence to deduce resulting probabilities.

Whenever a Bayesian Network estimates indirect measurements from direct measurements, it implements inference algorithms. Such inferences are computationally possible, thanks to the conditional probability relationships defined among the variables of the domain under analysis.

Bayesian networks are directed acyclic graph models for reasoning under uncertainty, where the nodes represent variables (discrete or continuous) and edges represent direct connections between them. These direct connections are often causal connections. Each node is associated with a probability function that takes a particular set of values for the input node variables, and gives as output, the probability (or probability distribution, if applicable) of the variable represented by the node. These probabilities (or probability distribution) are the relationships linking causes to effects between variables.

In $\mathrm{BN}$ modelling, any type of probability distribution function (PDF), such as uniform, normal, Poisson PDF, can be obtained with a directed edge between 2 nodes where the value domain of the input variable has been discretized. However, nonlinear functions of variables cannot be represented. A tool has been developed in Python 3 to manage the creation of the structure and the input of conditional probabilities. It relies to an adaptation of libpgm module.

In this work, all conditional probabilities, are empirically determined, based on observations and office occupant questionings. It has been defined for each node. They can be tuned from knowledge or from observations and questioning. Whenever the conditional probabilities of the appropriate nodes are set a BN model comes out.

\section{B. Hidden Markov Model}

A Hidden Markov Model (HMM) is a tool for representing probability distributions over sequences of observations and is in fact a special case of the more general BNs (Bayesian Networks). A HMM assumes the modeled system to be a Markov process, with an unobserved state sequence. In Markov models, the state is directly visible to the observer, and therefore, the state transition probabilities are the only parameters to be considered whereas, in a hidden Markov model, the state is not directly visible, but only outputs, dependent on the state, are visible. Each state has a probability distribution over the possible output tokens. Therefore the sequence of tokens generated by an HMM gives information about the sequence of states, and the challenge is to find out the parameters of such a model that maximizes the probability of setting the observations. The problem of learning the model parameters in an HMM can be solved by the forward backward Baum-Welch algorithm. Compared to a Bayesian network (BN), Hidden Markov Models (HMM) rely on nodes that represent system states, in other words the current state is dependent on the previous state.

By considering a set of states: $\left\{\right.$ Level $_{1}$, Level $_{2}, \ldots$ Level $\left._{N}\right\}$ Process moves from one state to another generating a sequence of states $:\left\{\right.$ occupancy $_{1}$, occupancy $_{2} \ldots$, occupancy $\left._{N}\right\}$ The probability of each subsequent state depends only on the previous state: $\mathrm{P}\left(\right.$ occupancy $_{k} \mid$ occupancy $_{1}$, occupancy $_{2} \ldots$ occupancy $\left._{k-1}\right)=$

$\mathrm{P}\left(\right.$ occupancy $\left._{k} \mid \operatorname{occupancy}_{(k-1)}\right)$.

To build a Markov model, the following probabilities have to be specified:

the transition probabilities 


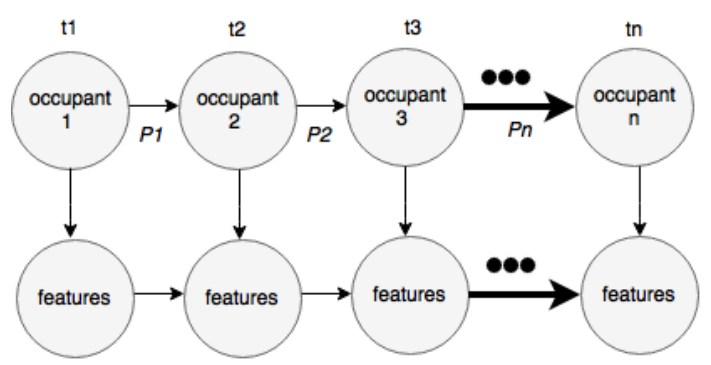

Figure 1. Hidden Markov Model(HMM) structure

$\mathrm{a}_{i j}=\mathrm{P}\left(\right.$ occupancy $\left.k \operatorname{occupancy}_{(k-1)}\right) \quad$ and initial probabilities $\pi_{i}=\mathrm{P}\left(\right.$ occupancy $\left._{i}\right)$ States are not visible, but each state randomly generates one of HMM observations $\left\{\right.$ features $_{1}$, features $s_{2} \ldots$ features $\left.{ }_{N}\right\}$.

Hidden Markov model has been defined by a matrix of transition probabilities $A=\left(\mathrm{a}_{i j}\right)$, $\mathrm{a}_{i j}=\mathrm{P}\left(\right.$ occupant $_{i} \mid$ occupant $\left._{j}\right)$, a matrix of observation probabilities $B=\left(\mathrm{b}_{i}\left(\right.\right.$ features $\left.\left._{m}\right)\right), \mathrm{b}_{i}\left(\right.$ features $_{m} \mid$ occupant $\left._{i}\right)$ and a vector of initial probabilities $\pi=(\pi)$, $\pi=\mathrm{P}\left(\right.$ occupant $\left._{i}\right)$. The model is represented by $\mathrm{HMM}=$ ( $A, B, \pi)$.

Important questions are raised before building the $\mathrm{BN}$ or HMM structure:

1) What knowledge domains are relevant?

2) What variables within these domains should be model?

3) What are the relationships between the variables?

4) What probabilities describe these relations?

In the next section the way of collecting the required knowledge has been proposed.

\section{CASE OF STUDY}

Let's consider a case of study to exemplify the capability of the approach for building HMM and BN structure. Three levels of occupancy have been chosen Low, Medium and High number of occupants. It is a good compromise for simplicity. While increasing the number of levels will complicate the table of conditional probability of $\mathrm{BN}$ structure and also the $\mathrm{HMM}=(A, B$, $\pi)$.

[15] indicates a correlation between the power consumption and the number of occupants in the office. For the sake of simplicity, three different cases have been considered, low consuming, medium consuming and high consuming, or $\mathrm{L}, \mathrm{M}$ and $\mathrm{H}$, respectively. It gives a probability table with 9 values (figure 2).

To collect the required information for conditional probabilities of $\mathrm{BN}$ and three questions have been proposed for the occupants in the office to determine the probability table for power consumption:

1) the schedule of occupants about arriving and leaving the office.

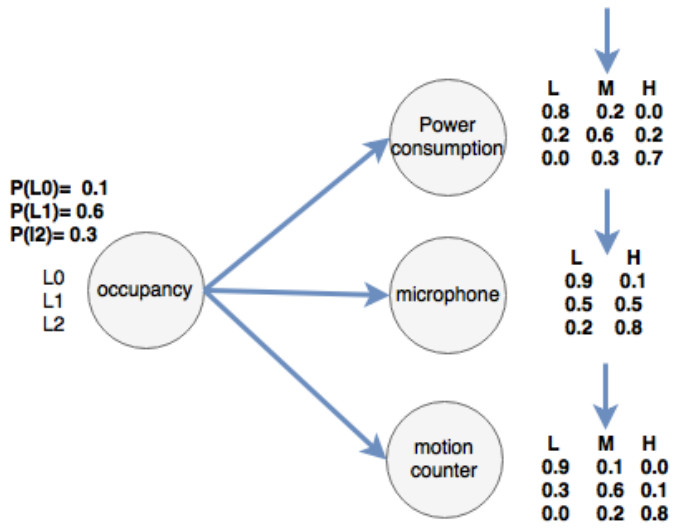

Figure 2. Bayesian network (BN) structure case of study

2) the average time for using the laptop during the working hours.

It is important to note that, the calendar of occupants could be used instead of the question, in order to facilitate the configuration of the $\mathrm{BN}$ structure.

Two different cases have been considered for the microphone Low acoustic pressure and high acoustic pressure or $\mathrm{L}$ and $\mathrm{H}$, respectively. It gives a table of probabilities with 6 values (figure 2).

The questions have been proposed to determine the probabilities table :

1) the kind of activities in the office (computer work, presentaton, skype meeting....... ).

2) the frequency of discussions between the colleagues during the working day.

While the probabilities table for motion counter have been suggested according to the general knowledge for three different cases low motion, medium motion and high motion, or $\mathrm{L}, \mathrm{M}$ and $\mathrm{H}$, respectively.

Considering the previous case of study, the occupancy is considered to be a hidden state (i.e 3 levels of occupants) and the most important features from the sensor network are observations as shown in Figure 3. To determine the matrix of output observation $B$, the same previous questions for the Power consumption, microphone and motion counter have been used. More probabilities have to be defined in HMM for transition matrix $A$. It needs more precise questions and observation of the presence of each occupant in the studied area.

HMM structure needs the same tables of probabilities as $\mathrm{BN}$ to get the observation matrix $B$, but in additions it needs the probabilities of each state taking into account the others states (considering the time) to get the transition Matrix $A$, see (figure 3.

It means that $\mathrm{BN}$ has less variables to be defined. The two approachesplotted BN and HMM, have been applied and the results comparision have been done in the next section. 


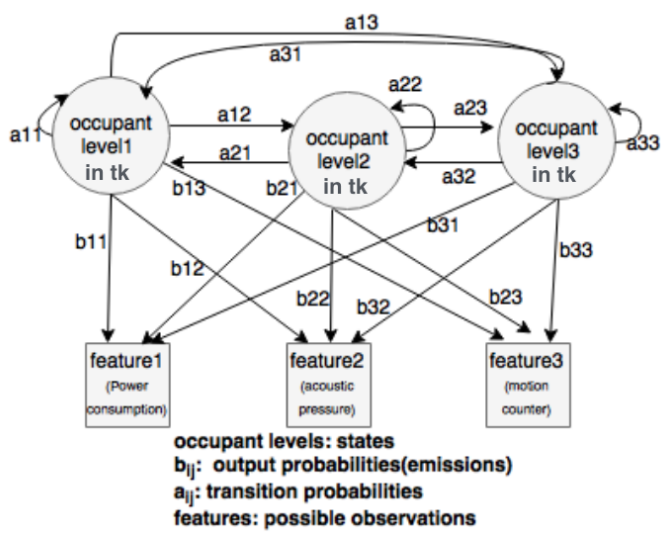

Figure 3. Hidden Markov Model (HMM) structure case of study

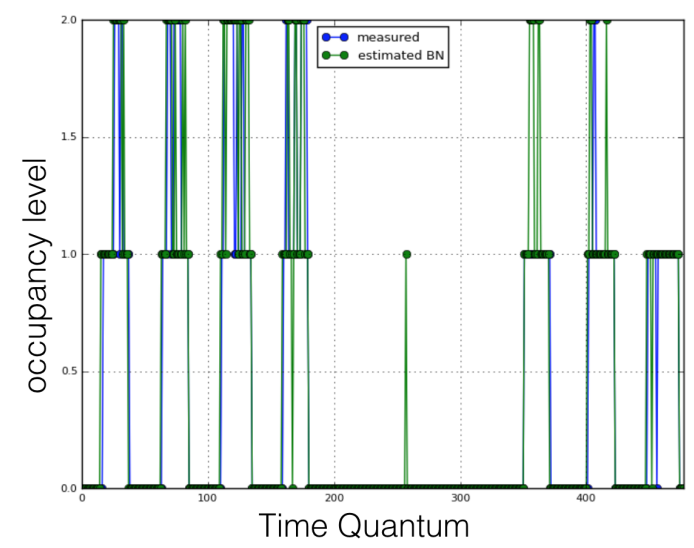

Figure 4. Occupancy estimation from BN

\section{RESULTS}

A dataset covering 10 days from 04-May-2015 to 13May-2015 have been used for building HMM and BN structure.

Figure 2 shows the results obtained from $\mathrm{BN}$ for three levels and 3 main features. Both actual and estimated occupancy profiles have been plotted in a graph with the number of occupants and relation to time (quantum time was 30 minutes). The accuracy achieved from $\mathrm{BN}$ was $91 \%$ (number of correctly estimated points divided by the total number of points), and the average error was 0.08 persons. Table II represents the average error values for each class of estimation.

\begin{tabular}{|c|c|c|c|}
\hline Classes & $\begin{array}{l}\text { Table II. BN ESTIM } \\
\text { Average error-BN }\end{array}$ & $\begin{array}{l}\text { TION RESULTS } \\
\text { accuracy-BN }\end{array}$ & Support \\
\hline Class1 & $97 \%$ & 0.001 & 400 \\
\hline Class2 & $84 \%$ & 0.2 & 170 \\
\hline Class3 & $79 \%$ & 0.5 & 54 \\
\hline average & $91 \%$ & 0.09 & 624 \\
\hline
\end{tabular}

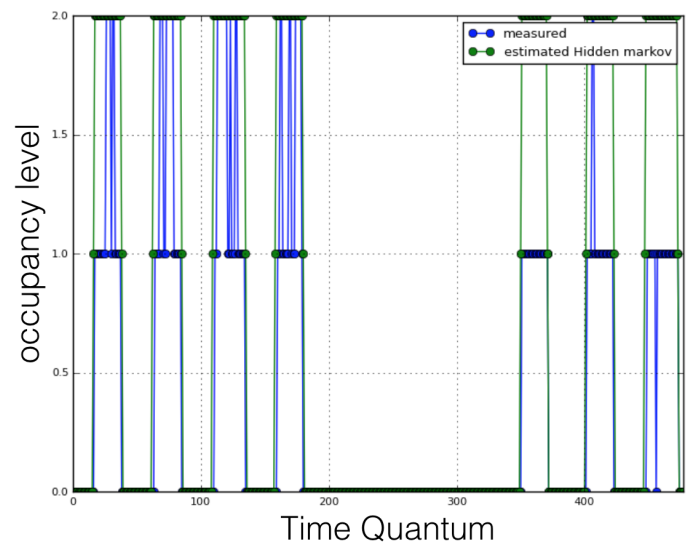

Figure 5. Occupancy estimation from HMM

Average error is more interesting than accuracy in the validation of occupancy estimation. Indeed, average error allows to distinguish each change in the estimated values while accuracy only considers the correctly estimated points.

Figure 5 represents the results obtained from the HMM, The accuracy achieved from HMM was $89 \%$ and the average error was 0.1 persons.

Better results have been obtained from $\mathrm{BN}$ comparing with HMM because of the complexity of HMM structure regarding the states relations of the $A$ matrix.

\begin{tabular}{cccc} 
c Classes & \multicolumn{2}{c}{ Table III. HMM ESTIM ATION RESULTS } & \\
Average error-HMM & accuracy-HMM & Support \\
\hline Class1 & $97 \%$ & 0.001 & 400 \\
Class2 & $82 \%$ & 0.25 & 170 \\
Class3 & $76 \%$ & 0.75 & 54 \\
average & $89 \%$ & 0.1 & 624
\end{tabular}

In order to improve HMM estimation, some knoweledge coming from DT have been applied by considering the if-then rules [15].

if motioncounter $>14$ or acousticpressure $>0.239$

or $\mathrm{CO}_{2}$ concentration $>1240$ then

increase the number of occupants by 1

\section{end if}

The accuracy achieved was $93 \%$ and the average error was 0.07 persons. About 45 parameters of thresholds have been extracted by the decision tree in estimating the occupant number [15]. Applying the algorithm C4.5 determines:more than 3 people will be in the office when motion counter threshold is more than 14 or when acoustic pressure from microphone is more than 0.239 $\mathrm{dB}$ or $\mathrm{CO}_{2}$ concentration is more than $1240 \mathrm{ppm}$. This knowledge is not represented precisely in HMM (little collecting information for the high levels of occupants), therefore it has been added to the estimation process and the number of occupants was increased by one.

A deeper analysis has been done regarding the accuracy 
and the average error for each day during the studied period, by applying the HMM and BN knowledge in table IV. The accuracy has a strong relation with the type of the day, in other words, it's very related to the actual level of occupancy in the studied area. Video cameras have been used to investigate what happened during the days with poor results. The highest accuracy is achieved during the non-working days $(08 / 05 / 15,09 / 05 / 15,10 / 05 / 15)$ with almost $100 \%$ while the lowest accuracy (75\% to $89 \%$ ) is met during the days of high level of occupancy with more than four persons $(05 / 05 / 15,06 / 05 / 15,07 / 05 / 15$, $12 / 05 / 15)$. For days where occupancy does not exceed 2 persons (13/05/15), an accuracy of $95 \%$ is obtained.

Table IV. ACCURACY AND AVERAGE ERROR OF EACH DAY DURING

\begin{tabular}{|c|c|c|c|c|}
\hline Day & accuracyBN & $\begin{array}{l}\text { STUDIED } \\
\text { errorBN }\end{array}$ & $\begin{array}{l}\text { EIOD } \\
\text { accuracyHMM }\end{array}$ & errorHMM \\
\hline $04 / 05 / 15$ & $86 \%$ & 0.13 & $80 \%$ & 0.19 \\
\hline $05 / 05 / 15$ & $79 \%$ & 0.2 & $78 \%$ & 0.26 \\
\hline $06 / 05 / 15$ & $77 \%$ & 0.22 & $73 \%$ & 0.22 \\
\hline $07 / 05 / 15$ & $87 \%$ & 0.125 & $78 \%$ & 0.2 \\
\hline $08 / 05 / 15$ & $100 \%$ & 0.0 & $100 \%$ & 0.0 \\
\hline $09 / 05 / 15$ & $98 \%$ & 0.02 & $99 \%$ & 0.01 \\
\hline $10 / 05 / 15$ & $100 \%$ & 0.0 & $100 \%$ & 0.0 \\
\hline $11 / 05 / 15$ & $83 \%$ & 0.16 & $86 \%$ & 0.1 \\
\hline $12 / 05 / 15$ & $89 \%$ & 0.1 & $93 \%$ & 0.06 \\
\hline $13 / 05 / 15$ & $94 \%$ & 0.06 & $95 \%$ & 0.05 \\
\hline
\end{tabular}

\section{CONCLUSION}

A non supervised learning approach has been proposed in this paper to estimate the number of occupants in an office setting. It results in a virtual sensor that relies on other sensors but with a superior performance. A human behaviour modelled by cause-effect and state relations has been obtained using Hidden Markov model and Bayesian network classification algorithms. Occupancy estimation using this knowledge gave a superior performance with an average estimation error of 0.1-0.08 occupants over ten days test period. Hidden Markov model and Bayesian network structures have been proposed in this paper to avoid the manual labeling of actual occupancy in a room for supervised learning approashes and solved the problem of using video cameras. The results were concluded that BN structure is less complicated than HMM, regarding the required states probabilities in HMM. This means improve the estimation process for more than 3 levels is less complicated in BN than HMM.

A non supervised learning procedure has been carried out using human knoweldge and questioning. This approch is very general because the structure is not assumed: it is discovered from the questioning and can be therefore extended to any room, thanks to expert knowledge.

In addition the estimation rules (if-then) which were extracted from the decision tree algorithm have been applied in the HMM, this knowledge improved the results with an average estimation error 0.07 occupants over a studied period. It has been combined with HMM structure to increase the accuracy of occupants estimation during the period of high level occupants resulting in a semi supervised learning approach. Obviously the same improvement could be done with $\mathrm{BN}$ estimation results.

Estimating the number of occupants is very interesting in many areas: simulating occupant behavior at design stage, predicting the number of occupants at energy management stage, dissociating building performance from usage at diagnosis stage, etc... The proposed approach can be extended to the estimation of occupant activities, which would be useful for developing interactive systems where suitable advice could be provided to occupants at appropriate times.

\section{REFERENCES}

[1] C. Roulet, R. Fritsch, J. Scartezzini, and P. Cretton, "Stochastic model of inhabitant behavior with regard to ventilation," Technical report, 1991.

[2] J. Page, D. Robinson, and J. Scartezzini, "Stochastic simulation of occupant presence and behaviour in buildings," Proc. Tenth Int. IBPSA Conf : Building Simulation, pp. 757-764, 2007.

[3] D. Robinson and F. Haldi, "Interactions with window openings by office occupants," Energy and Buildings, vol. 44, pp. 2378-2395, 2009.

[4] A. Kashif, J. Dugdale, and S. Ploix, "Simulating occupants' behaviour for energy waste reduction in dwellings: A multi agent methodology," Advances in Complex Systems, vol. 16, p. 37, 2013.

[5] K. K. A. B. O. Rune Vinther Andersenn, Jorn Toftum, "Survey of occupant behaviour and control of indoor environment in danish dwellings," Energy and Buildings, vol. 41, pp. 11-16, 2009.

[6] B. Mathieu, "Influence du comportement de l'occupant sur la performance energetique du batiment," Ph.D. dissertation, Universita de Toulouse, 2014.

[7] J. Langevin, j. Wen, and G. Patric, "Simulating the human-building interaction : Development and validation of an agent-based model of office occupant behaviours," Proc. Windsor Conference, Windsor, England, 2014.

[8] O. Nuria, G. Ashutosh, and H. Eric, "Layered representations for learning and inferring office activity from multiple sensory channels," 2004.

[9] M. Milenkovic and O. Amft, "Recognizing energy-related activities using sensors commonly installed in office buildings," Procedia Computer Science, vol. 19, pp. 669-677, 2013.

[10] Y. Agarwal, B. Balaji, R. Gupta, J. Lyles, M. Wei, and T. Weng, "Occupancy-driven energy management for smart building automation," in Proceedings of the 2nd ACM Workshop on Embedded Sensing Systems for Energy-Efficiency in Building. ACM, 2010, pp. 1-6.

[11] M. Philipose, K. P. Fishkin, M. Perkowitz, D. J. Patterson, D. Fox, H. Kautz, and D. Hahnel, "Inferring activities from interactions with objects," Pervasive Computing, IEEE, vol. 3, no. 4, pp. 5057, 2004.

[12] M. Milenkovic and O. Amft, "An opportunistic activity-sensing approach to save energy in office buildings," in Proceedings of the fourth international conference on Future energy systems. ACM, 2013, pp. 247-258.

[13] T. A. Nguyen and M. Aiello, "Beyond indoor presence monitoring with simple sensors." in PECCS, 2012, pp. 5-14.

[14] K. P. Lam, M. Höynck, B. Dong, B. Andrews, Y. shang Chiou, D. Benitez, and J. Choi, "Occupancy detection through an extensive environmental sensor network in an open-plan office building," in Proc. of Building Simulation 09, an IBPSA Conference, 2009.

[15] M. Amayri, A. Arora, S. Ploix, S. Bandhyopadyayc, Q.-D. Ngod, and V. R. Badarla, "Estimating occupancy in heterogeneous sensor environment estimating occupancy in heterogeneous sensor environment," Energy and Buildings, vol. 129, pp. 46-58, October 2016. 